\title{
Dynamic Field Mapping for Obtaining the Current Distribution in High-temperature Superconducting Tapes
}

\author{
Bertrand Dutoit, Joseph Duron, Svetlomir Stavrev and Francesco Grilli
}

\begin{abstract}
The magnetic field profile on the surface of $\mathrm{Bi}$ 2223/Ag tapes has been dynamically measured across the width of the samples. The experimental technique uses a Hall-probe array with 7 sensors connected to a multiple channel lock-in amplifier especially programmed for fast and synchronous data acquisition measurements. The speed of the system is high enough to measure real-time profiles with 7 probes and $50 \mathrm{~Hz}$ sine current through the sample. A numerical method to estimate the current distribution inside the tapes using the measured field profile data is proposed. The inverse problem has been solved using certain assumptions on the current distribution in the superconductor. Validation of the results has been done by comparison with finite element method simulations.
\end{abstract}

Index Terms-Bi-2223/Ag tapes, Hall probe arrays, lock-in technique, inverse problem, FEM modeling.

\section{INTRODUCTION}

$\mathrm{K}$ nowledge of the dynamic current distribution in the superconductor is a key element for understanding the conductor properties and for quality control purposes. It is also of great importance for the determination of the ac losses in high-temperature superconducting (HTS) tapes [1]. By measuring the magnetic field just above the conductor surface and after performing suitable data processing, a 2D map of the current distribution within the conductor can be calculated. This is the so-called 'inverse problem', whose solution is not straightforward since there are many possible current distributions that may result in a magnetic field profile corresponding to the measured one [2], [3].

We have employed the Hall-probe experimental technique [3] for the measurement of the magnetic field on the surface of sample HTS tapes because of the easier calibration of the Hall-probe sensors compared to magneto-optical measurements [4], even if the latter produce a higher spatial

Manuscript received October 5, 2004. This work has been supported by the Swiss Federal Office of Science and Education (OFES) through the Nationa Center for Competence in Research MaNEP (Materials with Novel Electronic Properties).

B. Dutoit, J. Duron, S. Stavrev and F. Grilli are with the Swiss Federal Institute of Technology - Lausanne, EPFL-IC-LANOS, 1015 Lausanne, Switzerland (tel.: +41-21-693-4689; fax: +41-21-693-6700; e-mail: Bertrand.Dutoit@epfl.ch). F. Grilli is now with the Los Alamos National Laboratory, MST-STC, Los Alamos, New Mexico 87545, United States. resolution map. Experimental Hall-sensor results for dc currents applied to HTS tapes have been already reported [5]. The existing techniques are, however, not truly dynamic, as the field mapping is not fast enough to respond to ac or fast ramp currents. In addition, the current distribution in ac varies along the cycle and only a truly dynamic measurement technique can provide information on the behaviour of the superconductor in ac conditions.

In this paper we present a newly developed experimental system that can measure dynamically at high sampling rate the magnetic field profile across mono or multifilamentary HTS tapes with frequency of the driving current up to $50 \mathrm{~Hz}$.

\section{DYNAMIC HALL-PROBE MEASUREMENTS}

\section{A. Experimental set-up}

For the data series acquisition, we have employed a high resolution 16-channel digital lock-in amplifier. The software lock-in has been integrated into a specially designed with LabView ${ }^{\circledR}$ complete measurement system with easily controlled frequency, amplitude, and driving current waveform. The update rate is $300 \mathrm{kHz}$ for the signal generation and $60 \mathrm{kHz}$ for the simultaneous sampling acquisition on the 16 channels with a 16-bit resolution. To avoid windowing in the lock-in calculation, the data block length should be chosen to have 8 to 16 complete cycles of the excitation frequency, thus ensuring good noise rejection. The experimental set-up is presented diagrammatically in Fig. 1.

The individual Hall probes are positioned $600 \mu \mathrm{m}$ apart along the sample width. In order to enhance the spatial resolution, a mobile Hall-probe array has been designed that can be mechanically moved laterally and longitudinally along the sample. The displacement is activated by step motors; each step corresponding to a $3.5 \mu \mathrm{m}$ move. The dynamic measurement is then repeated each time the sensors move by one step. A more detailed description of the experimental system with the mobile Hall-probe array and results from the corresponding measurements will be presented elsewhere [6].

A picture of the mobile head with 7 Hall-probes positioned on the surface of a sample at a distance of about $0.1 \mathrm{~mm}$ from the tape is shown in Fig. 2. 


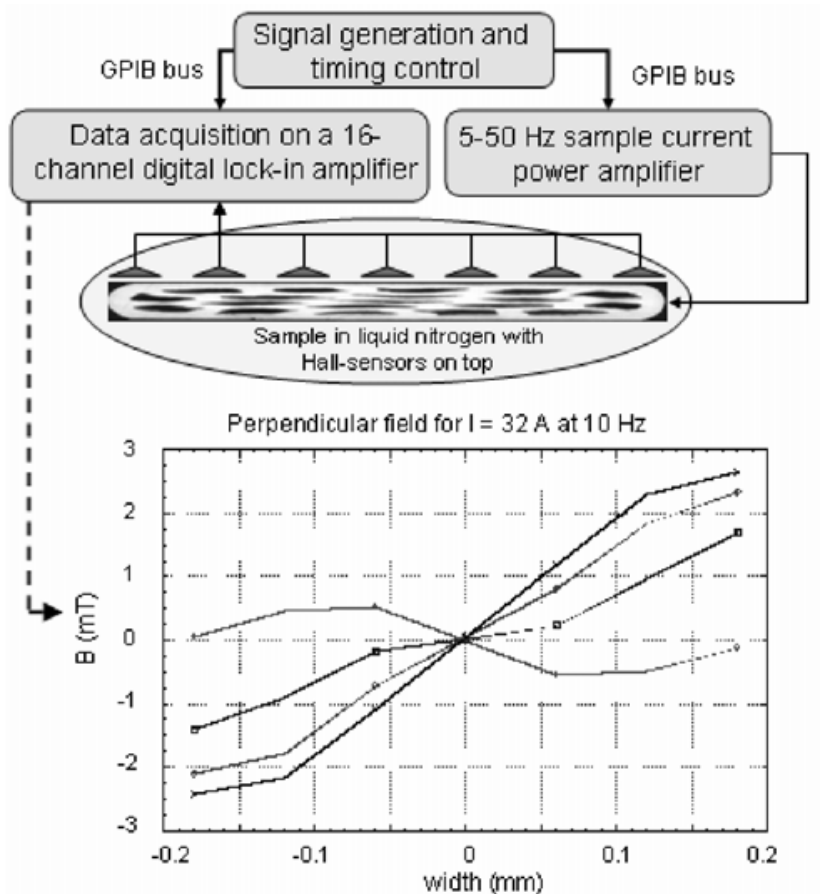

Fig. 1. Simplified diagram for the Hall-probe experimental set-up

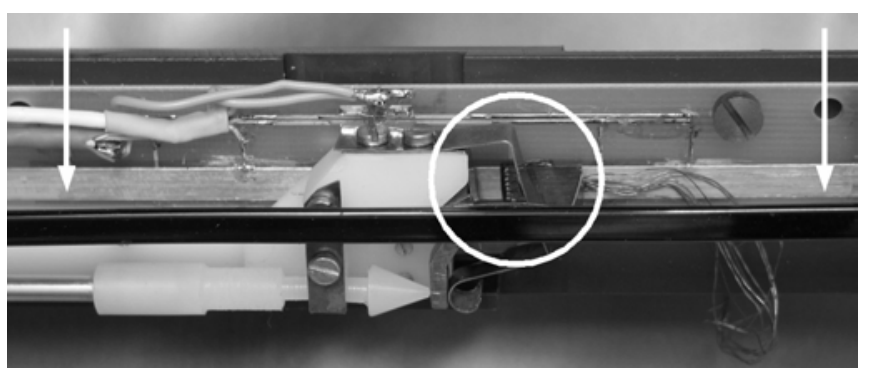

Fig. 2. Mobile head with 7 Hall probes positioned above the surface of a sample tape. The white circle indicates the position of the Hall-probe array and the arrows show the $10 \mathrm{~cm}$ sample.

The speed requirements for the data acquisition at $50 \mathrm{~Hz}$ are briefly outlined below. The $20 \mathrm{~ms}$ of the $50 \mathrm{~Hz}$ period are divided into 20 different measurements, in order to obtain truly dynamic field mapping. This means that the achieved frequency can be between 50 and $60 \mathrm{~Hz}$ if at least 20 samples are used to estimate the phase and the amplitude of the sine wave measured by each Hall probe, and for precision purposes 8 consecutive measurements are averaged.

The designed high-speed field-mapping system with 7 Hall probes at $50 \mathrm{~Hz}$, using a 16-channel digital lock-in amplifier, is able to simultaneously:

- generate the driving current through the superconductor at $625 \mathrm{ks} / \mathrm{s}$ (up to $50 \mathrm{~Hz}$, any waveform);

- generate the Hall excitation current at $625 \mathrm{Ks} / \mathrm{s}$ (6 to 10 $\mathrm{kHz}$ sine wave is used);

- acquire the data simultaneously on 8 channels at $156 \mathrm{ks} / \mathrm{s}$ per channel or on 16 channels at $78 \mathrm{ks} / \mathrm{s}$ per channel;

- compute the lock-in product on 8 or 16 channels;

- store the results in adapted files.
The measurement results presented below have been done with a fixed 7-Hall probe array and a monofilamentary $\mathrm{Bi}$ 2223/Ag tape with critical current $I_{c}=82 \mathrm{~A}$ (at $77 \mathrm{~K}$ ) and a fill-factor of $50 \%$, which gives a $J_{c}$ of $14 \mathrm{kA} / \mathrm{cm}^{2}$.

\section{B. Field measurement results}

An example of the evolution of the magnetic field in the vertical, or $y$-direction $\left(B_{y}\right)$, for one cycle at $50 \mathrm{~Hz}$, obtained with a series of consecutive measurements on the surface of the sample with a driving current of $70 \mathrm{~A}$ is shown in Fig. 3. It is interesting to note that even when the amplitude of the driving sine current is zero, for example at $\omega \mathrm{t}=\pi(\mathrm{t}=10 \mathrm{~ms}$ at $50 \mathrm{~Hz}$ ), the field profile is not zero, which is explained by the fact that at this moment the current within the superconductor flows with opposite directions in the external and internal filaments, and the current density is not zero, except in the center of the tape [7].

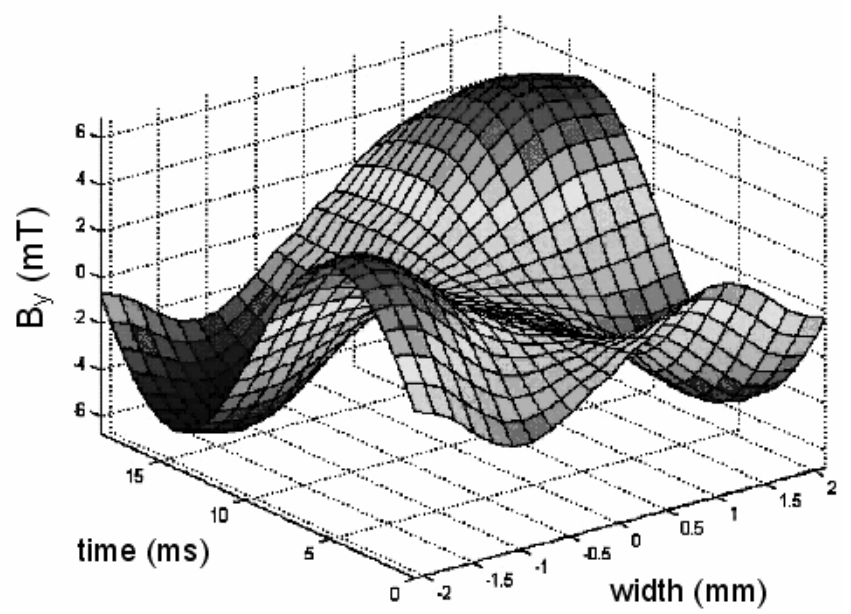

Fig. 3. Magnetic field profile (perpendicular component) along the width of a mono-filamentary tape for one cycle at $50 \mathrm{~Hz}$. The peak current is $70 \mathrm{~A}$.

\section{RECONSTRUCTION OF THE CURRENT DISTRIBUTION}

In order to obtain the map of the current density corresponding to the measured field profile in the y-direction, the inverse problem must be solved. In general, its solution is not unique since there may be several current distributions generating the measured $\mathrm{B}_{\mathrm{y}}$ profile. A method based on Ampere's theorem for calculating the current distribution in HTS tapes, using certain assumptions for the current density in the superconductor, has been especially developed for this purpose.

\section{A. Use of Ampere's integral law}

It is well known that a current flowing in an infinite straight wire (line current) produces a magnetic field linked to the current density by Ampere's integral law:

$$
\oint \frac{\vec{B}}{\mu_{0}} d \vec{l}=\int_{S} \vec{J} d S
$$

where $\vec{B}$ is the magnetic flux density, $\vec{J}$ is the current density in the wire, and $S$ is the wire's cross-sectional area. 
Applying Ampere's law to the case of line current with amplitude $I$, it is possible to find the magnetic field $\vec{B}$ produced by the current at a point with distance $r$ from the wire knowing the value of $I$, and vice-versa from the following relation:

$$
\vec{B}=\frac{\mu_{0}}{2 \pi} \frac{I}{r} \vec{e}_{\Theta}
$$

with $\vec{e}_{\Theta}$ a unit vector tangential to the circle of radius $r$ [7].

\section{B. Application to a superconducting tape}

Assuming the critical state model (CSM) in the superconductor, the tape can be divided into several sections of rectangular shape, where the current density is either $\pm J_{c}$ or zero, as shown in Fig. 4. A second approximation has been made for decreasing the calculation time - instead of making complete integrations on the sections, the current is considered to be concentrated at the gravity centre of a certain number of areas, into which each section is divided. If sufficiently large number of areas is taken into consideration, the approximation error is small. In the developed algorithm, the HTS tape is divided into $1000 \times 500(500,000)$ small elements along its width and thickness, respectively. Thus the tape can be approximated as a combination of infinite straight wires with radius much smaller than their length, each wire corresponding to one of the 500,000 small elements.

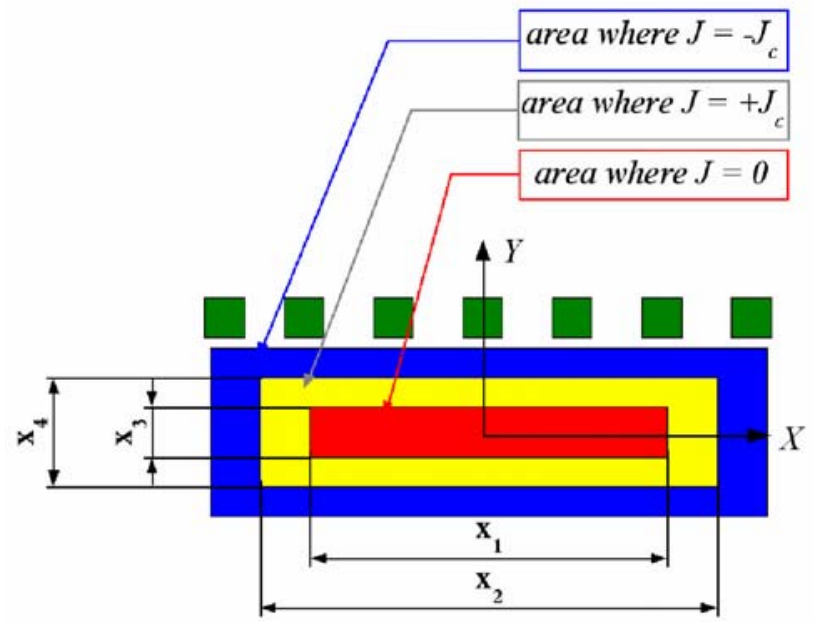

Fig. 4. Current density profile in a superconducting tape according to the critical state model. The square symbols represent the Hall probes.

Using Ampere's law, it is possible to calculate the contribution of each small element $d S$ in (1) and then to compare the computed and the measured magnetic field profiles. As the Hall probes give only the vertical component of the field $\mathrm{B}_{\mathrm{y}}$, Ampere's law has to be adapted to take into account this particularity:

$$
B_{y}\left(x_{c}, y_{c}\right)=\sum_{\text {area }} \frac{\mu_{0}}{2 \pi}|J| \int_{S} \frac{\operatorname{sign}(J)\left(x_{c}-x\right)}{\left(x_{c}-x\right)^{2}+\left(y_{c}-y\right)^{2}} d x d y
$$

where $x_{c}$ and $y_{c}$ are the coordinates of each probe. Thus, the magnetic field at the position of each Hall-probe is computed with (3) taking into account the contribution of the current in each small element with coordinates $x$ and $y$.

\section{Method for retrieving the current distribution in HTS tapes}

As shown above, using (1) and (2) it is possible to compute the magnetic field profile above the HTS tape given a certain current distribution inside the conductor. It is also possible to find the current distribution given the measured magnetic field profile, i.e. to find the solution of the inverse problem. For this purpose, a specially developed algorithm has been used for searching the width and the thickness of the rectangular sections with current density $\pm J_{c}$ or zero, such that the sum of the differences between the measured and calculated field profiles is minimized.

The developed optimization algorithm is based on the least squares method, applied to the minimization of the function

$$
f\left(x_{1}, x_{2}, x_{3}, x_{4}\right)=\sqrt{\sum_{\text {sensors }}\left(B_{y m}-B_{y c}\right)^{2}}
$$

where $\mathrm{B}_{\mathrm{ym}}$ is the measured magnetic field by each sensor, and $\mathrm{B}_{\mathrm{yc}}$ is the computed magnetic field at the location of each sensor with the summed contributions from all small elements. Two additional constraints have been added:

- the total current, obtained after integration of $J$ on the cross-section of the tape, must be equal to the current flowing through the tape (driving current);

- as no external magnetic field is applied, the geometry of the areas with $J= \pm J_{c}$ or zero must be symmetrical with respect to the $x$ - and $y$-axis because of the symmetry of the self-field.

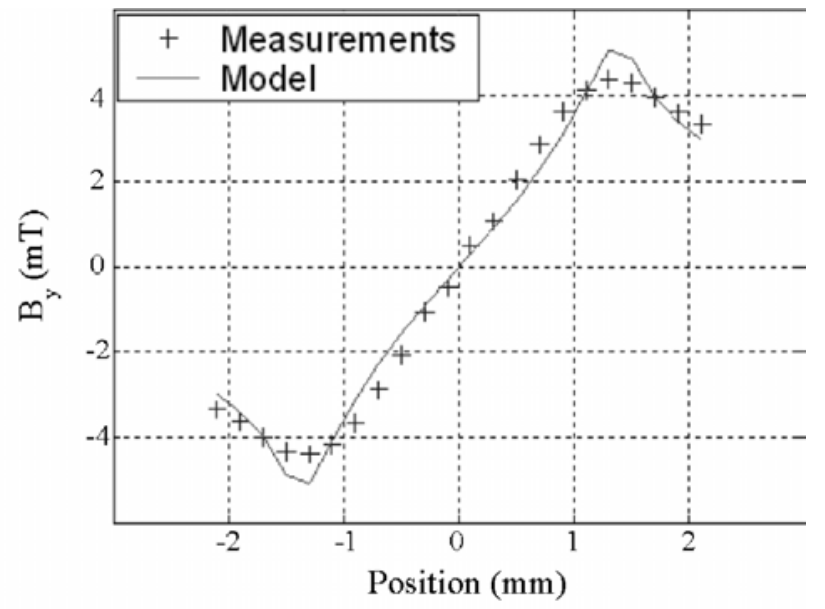

Fig. 5. Comparison between the magnetic field profiles - model and measurements with sine current of $70 \mathrm{~A}$ at $\omega \mathrm{t}=2 \pi$.

A result for applied sine current with amplitude of $70 \mathrm{~A}$ is given in Fig. 5 for $\omega t=2 \pi$. The plot gives a comparative example of the measured and calculated field profiles: the cross-symbols are the results obtained from the Hall-probe sensors and the solid line is the field corresponding to the reconstructed current distribution. Due to the strong assumptions, the optimization process is not perfect, but the model fits with a good approximation the experimental data. 


\section{COMPARISON WITH FEM SIMULATION RESULTS}

One of the advantages of the finite element method (FEM) is the fact that it gives the possibility to calculate precisely the current and field distribution inside superconducting wires with complex geometries [8], [9]. However, it is not possible to measure directly the current distribution inside the samples and to verify the FEM results even for superconductors with simple geometries. Therefore, for the purpose of validating both methods, a comparison has been made between the current distribution computed with the current-reconstruction model presented above, based on the Hall probe measurements, and the current distribution in a HTS tape computed with the electromagnetic software package Flux 3D [10]. Details about the FEM implementation, which uses a power-law relation of the type $E=E_{c}\left(J / J_{c}\right)^{n}$ for modeling of superconductors in Flux3D, can be found in [8]. The $J_{c}(B)$ and $n(B)$ dependence can also be implemented [9], but for the purpose of comparison with the current reconstruction method based on the CSM, in this paper constant $J_{c}=14 \mathrm{kA} / \mathrm{cm}^{2}$ and $n=21$ have been used in the FEM simulations.

a) $\omega t=3 \pi / 4$

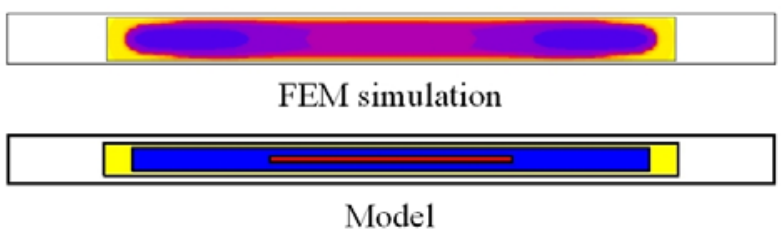

b) $\omega t=3 \pi / 2$

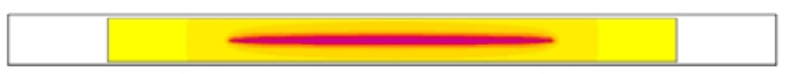

FEM simulation

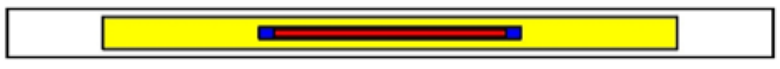

Model

c) $\omega t=7 \pi / 4$

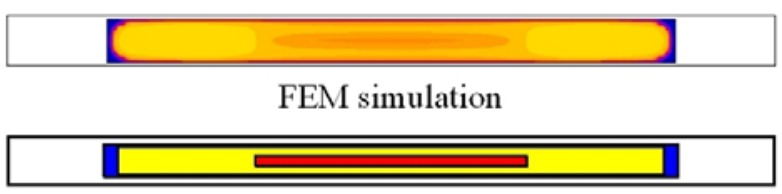

Model

d) $\omega t=5 \pi / 2$

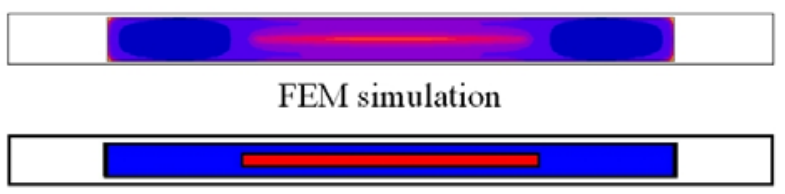

Model e) $\omega t=3 \pi$

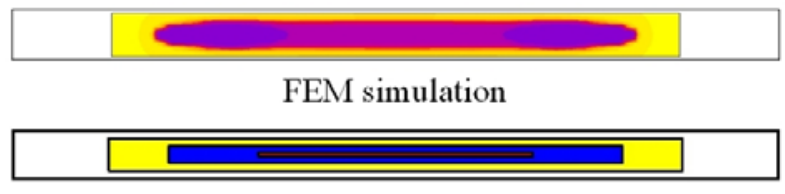

Model

Fig. 6. Comparison between the current density profiles obtained with FEM simulations and the reconstructed current distribution (model) for $\mathrm{I}=0.9 \mathrm{I}_{\mathrm{c}}$.

Figure 6 shows the current distributions, calculated at $\omega \mathrm{t}=$ $3 \pi / 4,3 \pi / 2,7 \pi / 4,5 \pi / 2$, and $3 \pi$ with the FEM software and the one obtained with the reconstruction method, based on the Hall probe measurements. As can be seen, the critical state approximation, even if a simple one, proves to be quite effective, and the reconstructed current distribution fits qualitatively well the finite element simulation results. It should be noted, however, that because of the assumption used in the current reconstruction method, the current density obtained in this way can take only values of $\pm J_{c}$ or zero, while the current density computed with the FEM may have any value between $-J_{c}$ and $+J_{c}$, and may even exceed locally the critical current density values[1], [8], [9].

\section{CONCLUSIONS AND FURTHER WORK}

The designed high-speed field-mapping system, using 16-channel digital lock-in amplifier, operating at frequencies up to $50 \mathrm{~Hz}$, with data acquisition rate of $78 \mathrm{ks} / \mathrm{s}$ per channel, provides truly dynamic, state-of-the-art performance. The magnetic field profile in perpendicular direction has been dynamically measured in ac conditions by placing an array of 7 Hall probes across the sample. In order to achieve higher lateral and longitudinal spatial resolution, the system has been upgraded with a new, mobile Hall-probe array.

The data from the measured field on the surface of $\mathrm{Bi}$ 2223/Ag tapes has been used in a specially developed inverse problem algorithm, based on CSM assumptions for finding the corresponding current distribution in the HTS tape. This method, mutually validated by comparison with results from FEM simulations, provides useful information for the current distribution inside the superconductor. The knowledge of the current distribution, obtained by this experimental technique, may be used for quality control purposes (e.g. detection of cracks along the length of the sample) and for gaining insight into the possible current variations in multifilamentary tapes with resistive barriers and/or non-uniform $J_{c}$ along the width.

The next steps for improving the proposed current reconstruction algorithm are: 1) to modify its geometry input in order to be used with multifilamentary tapes, and 2) to loosen the CSM assumption and to allow for non-uniform current distribution with possible variation of $J_{c}$ along the width of the conductors. In this way, the reconstructed current distribution needs not be symmetrical and cracks and other defects along the width and the length of HTS tapes could be detected. 


\section{REFERENCES}

[1] S. Stavrev, F. Grilli, B. Dutoit, I. Klutsch, E. Vinot , P. Tixador, G. Meunier, P. Skov-Hansen, and Jørn Hansen, "Numerical modeling of Bi-2223 multifilamentary tapes with position-dependent $\mathrm{J}_{\mathrm{c}}$ ", Physica $C$, Vol. 372-376, pp. 1800-1805, 2002

[2] H. Ochiai and S. Torii, "Application of inverse problem solution to the dynamic analysis of the internal current of bulk high-T-c superconductor", Physica C, vol. 357, pp. 777-780, 2001

[3] M. Masti, J. Lehtonen, and R. Mikkonen, "Accuracy of numerical analysis for Hall sensor magnetometer measurements", IEEE Trans. Appl. Supercon., vol. 13 (2), pp. 3671-3674, June 2003.

[4] G. Grimaldi, M. Bauer, H. Kinder, W. Prusseit, U. Gambardella, and S. Pace, "Magnetic imaging of YBCO coated conductors by Hall probes", Physica C, vol. 372, pp. 1009-1011, 2002

[5] M. Masti, J. Lehtonen, R. Perälä, W. Nah, and J. Kang, "Comparison of voltage-current characteristics of high quality Bi-2223 tapes with Hallsensor measurements and computed current density distributions", Physica C, vol. 401, pp. 155-159, 2004

[6] B. Dutoit, S. Stavrev, and J. Duron, unpublished

[7] H. Hauss and J. Melcher, in "Electromagnetic Fields and Energy", Prentice Hall, p. 19, 1989,

[8] S. Stavrev, F. Grilli, B. Dutoit, N. Nibbio, E. Vinot, I. Klutsch, G. Meunier, P. Tixador, Y. Yang, and E. Martinez., "Comparison of numerical methods for modeling of superconductors", IEEE Trans. Magnetics, vol. 38 (2), pp. 849-852, Mar. 2002.

[9] S. Stavrev, B. Dutoit, and F. Grilli, "Self-field and geometry effects in transport current applications of multifilamentary Bi-2223/Ag conductors ", IEEE Trans. Appl. Supercon., Vol. 13 (3), pp. 3807-3813, Sept. 2003

[10] Electromagnetic software package Flux3D, www.cedrat.com. 